\title{
Shunt Portocava e suas Implicações para o Transplante de Fígado: Uma Revisão Integrativa da Literatura
}

\author{
Olival Cirilo Lucena da Fonseca Neto ${ }^{1}$ (D), Maria Eduarda de Freitas Mesquita do Nascimento ${ }^{2}$ (D), \\ Lucas Loiola Ponte Albuquerque Ribeiro ${ }^{3}$ (1)
}

1. Hospital Universitário Oswaldo Cruz Rón Serviço de Cirurgia Geral e Transplante de Fígado, Recife (PE), Brasil.

2. Universidade de Pernambuco Rór Recife (PE), Brasil.

3. Universidade de Fortaleza Ró -

Fortaleza (CE), Brasil.

doi https://doi.org/10.53855/bjt.v25i1.426_pt

Autor correspondente:

olivallucena@gmail.com

Editora de Seção

Ilka Boin

Recebido

Dez. 14, 2021

Aprovado

Jan. 17, 2022

Conflito de interesse

Nada a declarar.

\section{Como Citar}

Fonseca Neto OCL, Nascimento MEFM, Ribeiro LLPA. Shunt Portocava e suas Implicações para o Transplante de Fígado: Uma Revisão Integrativa da Literatura.

BJT.2022; 25(01): e0322. https://doi.org/ 10.53855/bjt.v25i1.426_pt

eISSN

2764-1589

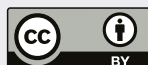

Resumo: Introdução: O clampeamento da veia porta pode gerar congestão venosa esplâncnica e aumento da hipertensão portal, causando liberação de mediadores inflamatórios no transplante hepático. Desenvolveu-se a técnica do bypass venoso, no início, para evitar-se congestão. Em 1968, foi descrita a técnica piggyback, e, em associação a ela, em 1993 e 1995, o shunt portocava temporário (SPCT), como alternativa para preservar o fluxo da veia cava e do sistema porta intraoperatório. Com a expansão do número de transplantes intervivos, foi desenvolvida uma adaptação da anastomose, ou shunt hemiportocava temporário (SHPCT). Objetivo: Esta revisão visou analisar a literatura encontrada nas bases de dados sobre SPCT e SHPCT no transplante hepático em adultos. Métodos: Revisão integrativa realizada por meio de buscas nas bases de dados PubMed, Biblioteca Eletrônica Científica Online (SciELO) e Literatura Latino-Americana e do Caribe em Ciências da Saúde (Lilacs), sendo selecionados 15 artigos publicados nos últimos 12 anos (2010-2021), após aplicação de critérios definidos pelos autores e inclusão de três trabalhos para referência histórica. Resultados: $\mathrm{O}$ aumento do tempo de cirurgia não parece ser desvantagem significativa que justifique contraindicação ao uso da anastomose portocava durante o procedimento. No transplante, há indicação de que o SPCT melhore a estabilidade hemodinâmica, com redução da infusão de concentrados de hemácias e plaquetas. Dados sobre níveis de aminotransferase, aspartato aminotransferase e índice internacional normalizado pós-operatórios foram conflitantes, sendo encontrada melhora na função renal, avaliada pela dosagem de creatinina. Os estudos demonstram relação do SPCT com diminuição de tempo de internamento hospitalar. Não foi possível chegar-se a uma conclusão a respeito dos impactos na sobrevida após o transplante. O SHPCT parece ser efetivo na diminuição do fluxo portal, servindo ao seu propósito de evitar o hiperfluxo, contudo não aparenta garantia absoluta de prevenir complicações. A maior limitação percebida foi a heterogeneidade dos estudos incluídos nesta revisão. Conclusão: Os estudos desenvolvidos apontam benefícios na estabilidade hemodinâmica e na função renal pós-operatória e menor tempo de internação. Não foi possível concluir acerca do impacto da técnica na lesão hepática, da sobrevida global nem do enxerto, pelas divergências na literatura.

Descritores: Derivação Portocava Cirúrgica; Transplante de Fígado; Veias Cavas.

\section{INTRODUÇÃO}

Durante a fase anepática do transplante de fígado, o clampeamento da veia porta do fígado do receptor pode gerar congestão venosa esplâncnica (anatomia representada na Fig. 1) e aumentar a hipertensão portal, o que leva ao edema intestinal e à disfunção da barreira da mucosa, com translocação bacteriana, endotoxemia e secreção de mediadores inflamatórios. ${ }^{1-3}$ Depois de desclampear a veia porta, esses fatores são 
liberados na circulação, podendo provocar vasodilatação sistêmica, hipotensão e síndrome da isquemia-reperfusão e, eventualmente, disfunção primária do enxerto. ${ }^{2,3}$

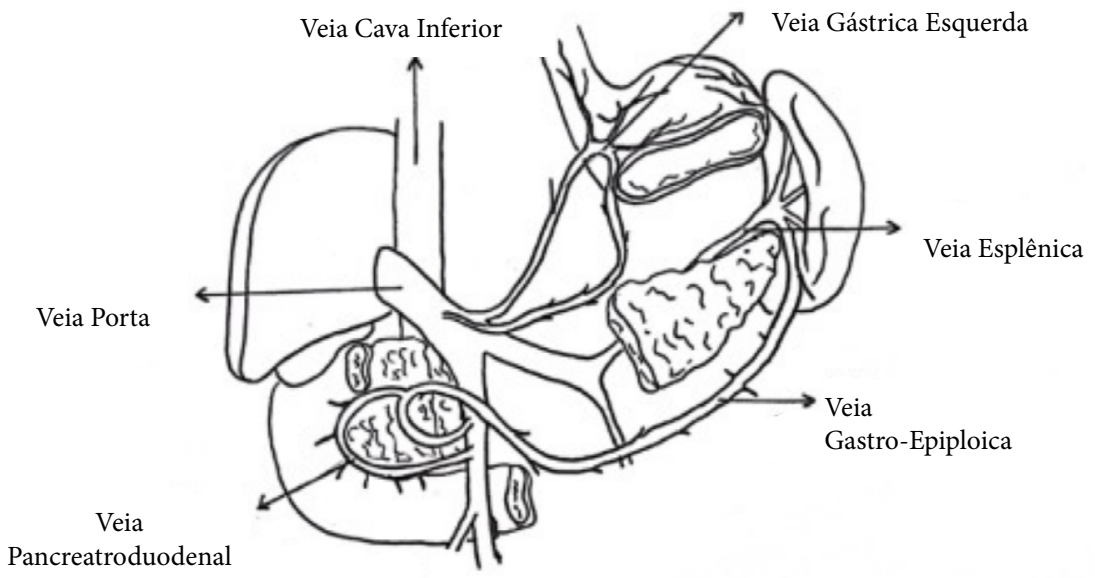

Figura 1. Representação da anatomia normal do sistema porta, demonstrando a interligação entre as drenagens venosas hepática, esplênica e intestinal.

Por isso, no início da história do transplante, desenvolveu-se a técnica do bypass venoso, para evitar a congestão venosa esplâncnica e sistêmica e outros efeitos deletérios causados pelo clampeamento da veia porta durante a fase anepática. Em 1968, foi descrito por Calne e Williams o transplante hepático com preservação da veia cava inferior, técnica que melhora o retorno venoso para o coração e diminui a congestão infradiafragmática pela preservação da veia cava inferior retro-hepática, melhorando a estabilidade hemodinâmica. ${ }^{4-9}$ Em associação a essa técnica, em 1993 e 1995, respectivamente, Belghiti et al. ${ }^{8}$ e Tzakis et al. ${ }^{10}$ popularizaram o shunt portocava temporário (SPCT) associado à técnica piggyback, como forma de preservar o fluxo da veia cava e do sistema porta durante todo o procedimento, principalmente em pacientes sem circulação colateral desenvolvida, como em casos de insuficiência hepática aguda, como representado na Fig. $2 .^{8-10}$

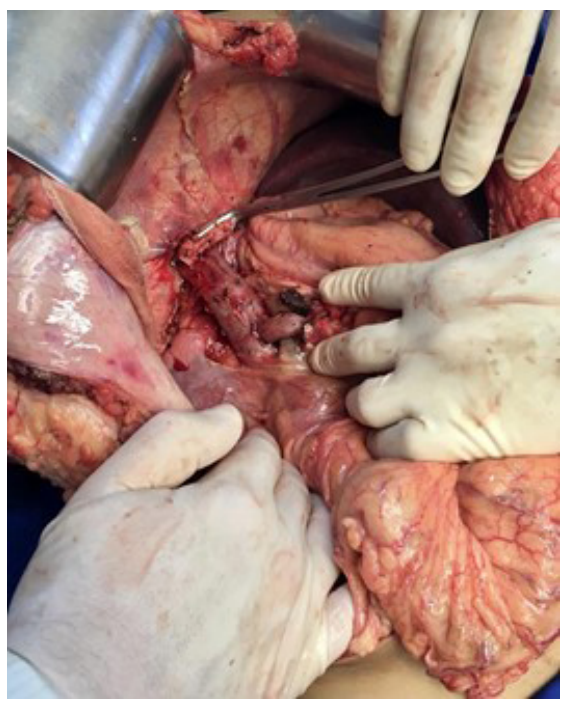

Figura 2. Imagem intraoperatória de uma anastomose portocava durante transplante de fígado.

Pela dificuldade de encontrar doadores suficientes e pela crescente demanda por transplante, o transplante intervivos ganhou cada vez mais espaço. ${ }^{10-12}$ Nesse contexto, o peso do enxerto tornou-se uma preocupação, pelo risco de desenvolvimento da síndrome small-for-size, cuja causa tem sido relacionada ao fluxo portal excessivo. ${ }^{11,12}$ Por isso, para evitar a lesão por elevação do fluxo portal, foi desenvolvida uma adaptação da técnica da anastomose portocava para modular o fluxo em transplantes 
intervivos, o chamado shunt hemiportocava temporário (SHPCT), ou anastomose hemiportocava.,11,13 Outras técnicas que podem ser utilizadas para modular o fluxo são esplenectomia, ligadura de artéria esplênica e shunt esplenorrenal. ${ }^{10,12}$

Apesar disso, os benefícios do SPCT permanecem controversos, com trabalhos mostrando resultados conflitantes. ${ }^{1,3,14,15}$ O objetivo desta revisão, portanto, foi fazer uma análise da literatura encontrada nas bases de dados sobre SPCT e SHPCT no transplante hepático em adultos.

\section{METODOLOGIA}

Trata-se de uma revisão integrativa da literatura disponível sobre as implicações da técnica SPCT no transplante hepático. Foi realizada uma busca nas bases de dados PubMed, Biblioteca Eletrônica Científica Online (SciELO) e Literatura LatinoAmericana e do Caribe em Ciências da Saúde (Lilacs), com os descritores anastomose portocava e transplante de fígado, e seus equivalentes em inglês, aplicando-se o filtro humanos. Na base PubMed, encontraram-se 63 resultados para o período determinado (2010-2021), e, após a leitura dos títulos e resumos, foram excluídos artigos que não abordaram diretamente o transplante hepático, trabalhos com animais, estudos pediátricos e um artigo que descreveu a técnica como ponte para transplante, e não como abordagem intraoperatória durante transplante hepático. Não foi achado nenhum artigo nas bases SciELO e Lilacs.

Ademais, realizou-se busca por shunt hemiportocava e seu equivalente em inglês nas bases de dados supracitadas, sendo encontrados sete resultados na base PubMed, dos quais quatro foram excluídos, três por não abordarem diretamente shunt hemiportocava e um por duplicidade. Não foram encontrados artigos nas bases SciELO e Lilacs. Além disso, incluíramse manualmente três trabalhos, por serem as publicações que primeiramente descreveram a técnica de SPCT no transplante hepático, por fins históricos.

Ao final, foram incluídos 18 artigos nesta revisão integrativa, com o processo de seleção descrito na Fig. 3.

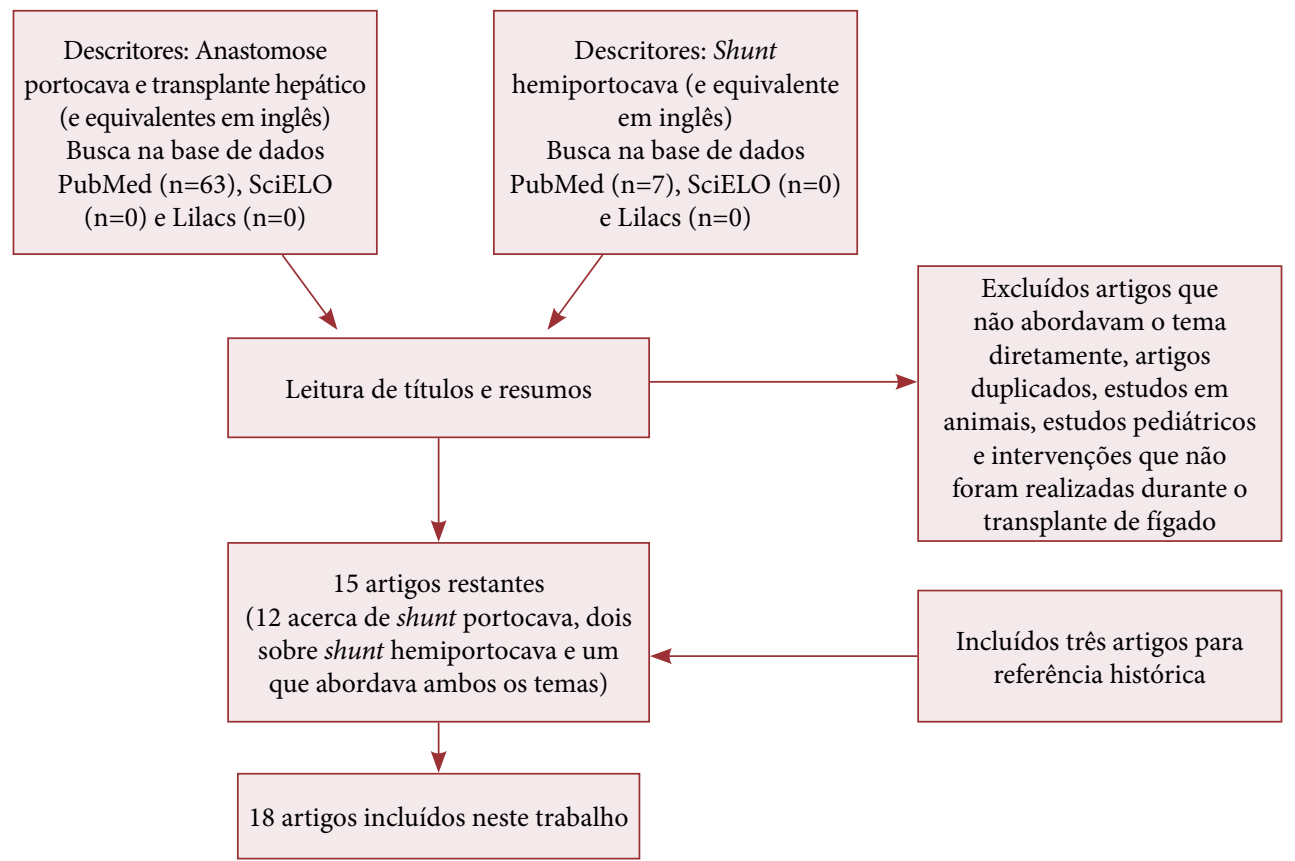

Figura 3. Representação do processo de seleção de artigos para esta revisão.

Para analisar a utilização da anastomose portocava temporária no transplante hepático, foram analisados os seguintes aspectos: tempo de cirurgia; estabilidade hemodinâmica, avaliada pelo número de concentrados de hemácias e de plasma fresco congelado, bem como pelo uso de vasopressores; infecção pós-operatória; lesão hepática, avaliada mediante a aminotransferase alanino aminotransferase (ALT), o aspartato aminotransferase (AST) e o índice internacional normalizado (INR) pós-operatórios; função renal, avaliada pela creatinina sérica; tempo de internamento; e sobrevida global e do enxerto. 


\section{RESULTADOS E DISCUSSÃO}

\section{Tempo de cirurgia}

O aumento do tempo de cirurgia para a realização do SPCT foi uma preocupação levantada por alguns autores, entretanto, na metanálise feita por Pratschke et al., ${ }^{14}$ o tempo de procedimento revelou-se menor do que o do grupo sem SPCT. O mesmo foi visto em estudo retrospectivo de Nacif et al., ${ }^{16}$ enquanto outros autores relataram que não houve diferença. ${ }^{2,7,15,17}$ Segundo Belghiti et al., ${ }^{8}$ o tempo médio para a realização do shunt é de 9 minutos, e para Faitot et al. ${ }^{9}$ o tempo varia entre 5 e 15 minutos.

Apenas dois estudos, de Rayar et al. $^{3}$ e Weniger et al., ${ }^{18}$ encontraram aumento do tempo cirúrgico no grupo submetido a SPCT. Nos casos em que se verificou aumento da fase anepática no grupo SPCT, como no estudo prospectivo conduzido por Son et al., ${ }^{17}$ não houve impacto na sensibilidade anestésica nem na função cognitiva pós-operatória.

Portanto, o aumento do tempo de cirurgia não parece ser desvantagem significativa que justifique contraindicação ao uso da anastomose portocava durante o transplante hepático.

\section{Estabilidade hemodinâmica}

Na metanálise incluída neste trabalho, foi identificada redução significativa no número de transfusões de concentrados de hemácias no grupo submetido a SPCT, o que pode sugerir melhor estabilidade hemodinâmica por menor perda volêmica, ${ }^{7}$ entretanto não houve diferença na transfusão de plasma fresco congelado entre os pacientes submetidos a SPCT e os não submetidos. ${ }^{7}$

Pratschke et al., ${ }^{14} \mathrm{em}$ sua análise retrospectiva, encontraram redução significativa no uso de vasopressores nos pacientes submetidos a SPCT, enquanto o trabalho de Ghinolfi et al. ${ }^{15}$ verificou tendência não significativa a maior uso de vasopressores em pacientes não submetidos a shunt, tendo estes recebido maiores doses de fenilefrina, mas sem diferença significativa nas doses necessárias de epinefrina e norepinefrina. Todavia, Weniger et al. ${ }^{18}$ não constataram diferenças no uso de norepinefrina entre os grupos.

Avaliando-se os dados disponíveis, há indicação de que a realização de SPCT durante o transplante hepático melhore a estabilidade hemodinâmica dos pacientes, mas ainda não há consenso na literatura sobre isso.

\section{Infecção pós-operatória}

O clampeamento da veia porta durante o procedimento foi associado a aumento do edema intestinal e translocação bacteriana. ${ }^{1,3,7,14}$ Apesar da fisiopatologia descrita em diversos trabalhos, apenas um estudo investigou se a translocação descrita resultou em aumento de infecção pós-operatória, comparando grupos com SPCT e uso de meropenem, SPCT e uso de meropenem acrescido de vancomicina e grupo sem SPCT. ${ }^{18}$

A hipótese, entretanto, mostrou-se nula, não havendo diferença entre os três grupos no que se refere à incidência geral de infecções e a infecções por gram-negativos, verificando-se apenas tendência a maiores infecções por gram-positivos no grupo SPCT e uso de meropenem. Esse dado, somado ao achado de melhor sobrevida no grupo SPCT e uso de meropenem e vancomicina, parece sugerir que a inclusão da vancomicina na antibioticoprofilaxia seria benéfica. ${ }^{18}$

\section{Lesão hepática}

Diversos estudos analisaram lesão hepática pelas medidas de ALT e AST pós-transplante. Dois estudos identificaram menores níveis de AST em pacientes submetidos a SPCT (em um deles, viu-se redução não significativa estatisticamente), sugerindo menor agressão aos hepatócitos, entretanto não houve diferença nos níveis de ALT, ${ }^{7,15}$ enquanto outro trabalho verificou redução em ambos. ${ }^{14}$ Sobre os tempos de protrombina e INR, os dois artigos que analisaram essas variáveis tiveram resultados conflitantes, com um deles sugerindo melhora nos casos em que foi realizado SPCT, e outro sem diferença entre os grupos. ${ }^{3,15}$

\section{Função renal}

A metanálise de Pratschke et al. ${ }^{7}$ encontrou diminuição nos valores de creatinina no pós-operatório dos pacientes submetidos a SPCT em comparação ao controle em todos os estudos incluídos, exceto em um, que não verificou variações significativas. No trabalho de Ghinolfi et al., ${ }^{15}$ o benefício nos valores de creatinina só foi significativo nas primeiras $72 \mathrm{~h}$ após o procedimento, e, depois desse período, os valores de ambos os grupos se tornaram similares. 
Com base nesses dados, o SPCT parece melhorar a perfusão renal, como sugerido pela fisiopatologia, levando à melhor função renal pós-operatória.

\section{Tempo de internamento hospitalar}

As duas metanálises incluídas neste estudo identificaram menor tempo de internamento na unidade de terapia intensiva e de internamento total em pacientes submetidos a SPCT. Em uma delas, a diferença foi estatisticamente significativa. ${ }^{5,7}$ Os trabalhos de Rayar et al. ${ }^{3}$ e Ghinolfi et al., ${ }^{15}$ entretanto, encontraram resultados dissonantes, sem diferença no tempo de internamento entre pacientes submetidos ou não ao shunt temporário, sendo ambos análises retrospectivas. Como as metanálises possuem maior nível de evidência e análise metodológica mais apurada, acredita-se que o SPCT pode estar relacionado à redução do tempo de internação.

\section{Sobrevida do enxerto e sobrevida global}

Dois trabalhos não encontraram diferença entre a sobrevida global e a do enxerto comparando grupos com e sem SPCT, ${ }^{1,3}$ entretanto outros dois estudos encontraram melhor sobrevida global entre pacientes maiores de 70 anos quando submetidos a SPCT.,16

Analisando a sobrevida do enxerto em três meses, dois trabalhos demonstraram melhor sobrevida em pacientes submetidos à anastomose portocava temporária. ${ }^{3,5}$ Esse benefício parece estar presente sobretudo nos enxertos advindos de doadores de critério estendido, que parecem ser especialmente suscetíveis à síndrome da isquemia-reperfusão. ${ }^{3}$

Como os estudos apresentam resultados conflitantes, ainda não foi possível concluir sobre a relação da anastomose portocava nem acerca da sobrevida após transplante de fígado.

\section{Hemiportocava no transplante intervivos}

O SHPCT parece ser efetivo na diminuição do fluxo portal, servindo ao seu propósito de evitar o hiperfluxo, o principal componente da síndrome small-for-size, que se manifesta com colestase, coagulopatia, hipertensão portal e encefalopatia, podendo levar à morte. ${ }^{11,13}$ Todavia, não parece ser garantia absoluta na prevenção da síndrome, que foi identificada mesmo entre pacientes submetidos a SHPCT. ${ }^{11}$

A principal complicação está relacionada à redução excessiva do fluxo, causando a chamada síndrome do roubo portal, a qual se manifesta com encefalopatia e falha na regeneração do enxerto. ${ }^{11,12} \mathrm{O}$ principal fator de risco para o seu desenvolvimento é a existência de grandes shunts espontâneos não conhecidos, mas também pode ser causado pelo shunt construído. ${ }^{12}$

Para evitar os extremos e assegurar um fluxo adequado, foram propostos como parâmetros o diâmetro da anastomose e o gradiente de pressão da veia cava, porém eles ainda carecem de validação e padronização. ${ }^{11,12}$ A necessidade de fechar o shunt é controversa. Alguns autores estimulam a avaliação individual dos casos e argumentam contra a necessidade de fechar rotineiramente o shunt em todos os pacientes, ${ }^{11}$ no entanto outros autores recomendam o fechamento do shunt para prevenir a encefalopatia. ${ }^{13}$ De qualquer maneira, é preciso levar em consideração que há possibilidade de fechamento espontâneo do shunt, com um trabalho descrevendo tal ocorrência em ${ }^{2 / 3}$ dos pacientes. ${ }^{13}$

\section{Limitações}

Os artigos analisados incluíram populações heterogêneas e metodologias diversas, a maioria retrospectiva, avaliando diferentes variáveis, impedindo a comparação direta, especialmente no que diz respeito ao SHPCT, cujo número de publicações foi bastante limitado. Foram identificados déficits na literatura acerca do tema na língua portuguesa, com apenas um trabalho sobre SPCT no Brasil, indicando a necessidade de novos estudos.

Ressalta-se a importância de desenvolver-se mais estudos concernentes ao tema, especialmente com metodologias que gerem níveis mais altos de evidência, como estudos prospectivos controlados, revisões sistemáticas e metanálises. Além disso, recomenda-se a feitura de estudos com população-alvo nacional, para melhor análise dos impactos de tal procedimento no Brasil, tornando possível compreender as especificidades e o perfil da população brasileira.

\section{CONCLUSÃO}

O SPCT com preservação da veia cava é uma técnica para preservar o fluxo da veia cava e do sistema porta durante o transplante hepático. Os estudos desenvolvidos sobre o tema apontam para benefícios na estabilidade hemodinâmica, função renal pós-operatória e redução do tempo de internação. 
Não foi possível concluir sobre o impacto da técnica na lesão de isquemia-reperfusão hepática nem na sobrevida global do paciente e do enxerto pelas relevantes divergências na literatura. Já no transplante intervivos, o SPHCT pode ser utilizado para prevenção da síndrome small-for-size, mas, pela escassez de dados, ainda não é possível determinar a necessidade posterior de fechamento do shunt.

\section{CONTRIBUIÇÃO DOS AUTORES}

Contribuições científicas e intelectuais substantivas para o estudo: Fonseca Neto OCL, Nascimento MEFM e Ribeiro LLPA; Concepção e projeto: Fonseca Neto OCL e Nascimento MEFM; Análise e interpretação de dados: Nascimento MEFM e Ribeiro LLPA; Escrita do manuscrito: Nascimento MEFM e Ribeiro LLPA; Aprovação final: Fonseca Neto OCL.

\section{DISPONIBILIDADE DE DADOS DE PESQUISA}

Não se aplica.

\section{FINANCIAMENTO}

Não se aplica.

\section{AGRADECIMENTOS}

Não se aplica.

\section{REFERÊNCIAS}

1. Pietersen LC, Sarton E, Alwayn I, Lam HD, Putter H, van Hoek B, et al. Impact of temporary portocaval shunting and initial arterial reperfusion in orthotopic liver transplantation. Liver Transpl. 2019;25(11):1690-9. https://doi.org/10.1002/lt.25592

2. Kim JD, Choi DL. Beneficial impact of temporary portocaval shunt in living-donor liver transplantation with a difficult total hepatectomy. Transplant Proc. 2015;47(3):694-9. https://doi.org/10.1016/j.transproceed.2014.12.036

3. Rayar M, Levi Sandri GB, Cusumano C, Locher C, Houssel-Debry P, Camus C, et al. Benefits of temporary portocaval shunt during orthotopic liver transplantation with vena cava preservation: a propensity score analysis. Liver Transpl. 2017;23(2):174-83. https://doi.org/10.1002/lt.24650

4. Calne RY, Williams R. Liver transplantation in man. I. Observations on technique and organization in five cases. Br Med J. 1968;4:535-40. https://doi.org/10.1136/bmj.4.5630.535

5. Nacif LS, Zanini LY, Sartori VF, Kim V, Rocha-Santos V, Andraus W, et al. Intraoperative surgical portosystemic shunt in liver transplantation: systematic review and meta-analysis. Ann Transplant. 2018;23:721-32. https://doi.org/10.12659/ AOT.911435

6. Addeo P, Locicero A, Faitot F, Bachellier P. Temporary right portocaval shunt during piggyback liver transplantation. World J Surg. 2019;43(10):2612-5. https://doi.org/10.1007/s00268-019-05042-7

7. Pratschke S, Rauch A, Albertsmeier M, Rentsch M, Kirschneck M, Andrassy J, et al. Temporary intraoperative porto-caval shunts in piggy-back liver transplantation reduce intraoperative blood loss and improve postoperative transaminases and renal function: a meta-analysis. World J Surg. 2016;40(12):2988-98. https://doi.org/10.1007/s00268-016-3656-1

8. Belghiti J, Noun R, Sauvanet A. Temporary portocaval anastomosis with preservation of caval flow during orthotopic liver transplantation. Am J Surg. 1995;169(2):277-9. https://doi.org/10.1016/S0002-9610(99)80151-2

9. Faitot F, Addeo P, Besch C, Michard B, Oncioiu C, Ellero B, et al. Passive mesenterico-saphenous shunt: An alternative to portocaval anastomosis for tailored portal decompression during liver transplantation. Surgery. 2019;165(5):970-7. https:// doi.org/10.1016/j.surg.2018.10.036

10. Tzakis AG, Reyes J, Nour B, Marino IR, Todo S, Starzl TE. Temporary end to side portacaval shunt in orthotopic hepatic transplantation in humans. Surg Gynecol Obstet [Internet]. 1993 [cited 2021 May 23];176(2):180-2. Available from: https:// www.ncbi.nlm.nih.gov/pmc/articles/PMC2729161/ 
11. Botha JF, Langnas AN, Campos BD, Grant WJ, Freise CE, Ascher NL, et al. Left lobe adult-to-adult living donor liver transplantation: small grafts and hemiportocaval shunts in the prevention of small-for-size syndrome. Liver Transpl. 2010;16(5):649-57. https://doi.org/10.1002/1t.22043

12. Kinaci E, Kayaalp C. Portosystemic shunts for "too small-for-size syndrome" after liver transplantation: a systematic review. World J Surg. 2016;40(8):1932-40. https://doi.org/10.1007/s00268-016-3518-X

13. Goja S, Srivastava M, Singh M, Sinha R, Roy R, Mahabaleshwar V, et al. Is portal inflow modulation always necessary for successful utilization of small volume living donor liver grafts? Liver Transpl. 2020;26(2):304-5. https://doi.org/10.1002/ lt. 25670

14. Pratschke S, Meimarakis G, Bruns CJ, Kaspar M, Prix N, Zachoval R, et al. Temporary intraoperative porto-caval shunt: useless or beneficial in piggy back liver transplantation? Transpl Int. 2013;26(1):90-8. https://doi.org/10.1111/tri.12007

15. Ghinolfi D, Martí J, Rodríguez-Laiz G, Sturdevant M, Iyer K, Bassi D, et al. The beneficial impact of temporary porto-caval shunt in orthotopic liver transplantation: a single center analysis. Transpl Int. 2011;24(3):243-50. https://doi.org/10.1111/ j.1432-2277.2010.01168.x

16. Nacif LS, Zanini LY, Costa dos Santos JP, Pereira JM, Pinheiro RS, Rocha-Santos V, et al. Intraoperative temporary portocaval shunt in liver transplant. Transplant Proc. 2020;52(5):1314-7. https://doi.org/10.1016/j.transproceed.2020.02.074

17. Son YG, Byun SH, Kim JH. The anhepatic phase extended by temporary portocaval shunt does not affect anesthetic sensitivity and postoperative cognitive function: a case-control study. Medicine (Baltimore). 2016;95(49):e5654. https://doi. org/10.1097/MD.0000000000005654

18. Weniger M, Andrassy J, Weig T, Grabein B, Crispin A, Rentsch M, et al. Temporary intra-operative portocaval shunts, postoperative infections, and mid-term survival after cava-sparing liver transplantation. Surg Infect (Larchmt). 2017;18(7):8039. https://doi.org/10.1089/sur.2017.036 